\title{
Diretrizes para o design de interfaces de Ambientes Virtuais de Aprendizagem voltadas a usuários com baixa visão
}

\author{
Interface design guidelines for visually impaired \\ Learning Management Systems users
}

acessibilidade, baixa visão, design de interfaces, ambientes virtuais de aprendizagem accessibility, visual impairment, interface design, learning management system
Esta pesquisa descreve o processo de elaboração de um conjunto de 23 diretrizes para design de interfaces de Ambientes Virtuais de Aprendizagem, voltadas aos estudantes com baixa visão. As diretrizes foram elaboradas de forma colaborativa e centrada no usuário, contando com a colaboração de 12 estudantes com baixa visão (de níveis diversos), 3 especialistas em acessibilidade e 5 desenvolvedores e designers de web sites. A partir do levantamento de necessidades dos estudantes, os especialistas em acessibilidade geraram um protocolo de navegação para os estudantes com baixa visão, o que permitiu identificar suas dificuldades. A partir disso, o conjunto de diretrizes foi sucessivamente refinado de forma colaborativa entre os especialistas em acessibilidade e os designers e programadores, resultando num conjunto que é apresentado ao final deste artigo. Ainda que a maioria das diretrizes listadas nesta pesquisa não representem novidade em termos de design de interfaces, o fato de elas terem sido agrupadas em torno das necessidades dos usuários com baixa visão representa um avanço, pois facilita o uso destas diretrizes por desenvolvedores que não são especialistas no assunto.

This paper describes the process of elaborating a set of 23 guidelines for the design of LMS interfaces, aimed at students with visual impairment. The guidelines were developed in a collaborative and user-centered manner, with the collaboration of 12 students with visual impairment (of different levels), 3 specialists in accessibility and 5 web developers and designers. From the survey of students' needs, the accessibility specialists generated a navigation protocol for students with low vision, which allowed them to identify their difficulties. From this, the set of guidelines was successively refined in a collaborative manner between accessibility specialists and designers and programmers, resulting in the set presented at the end of this paper. Although most of the guidelines listed in this research are not new in terms of interface design, the fact that they have been grouped around the needs of users with visual impairment is an advance, as it facilitates the use of these guidelines by developers who are not subject matter experts. 


\section{Introdução}

A popularização das tecnologias digitais de comunicação e do acesso à internet refletiu positivamente na distribuição de conteúdo das mais diversas áreas, aumentando a inteligência coletiva. Nesse contexto, o acesso à informação pode representar a possibilidade de melhoria das condições de vida, por meio da mobilidade social, pois as interações acadêmicas e profissionais são mediadas pelas tecnologias digitais. Quando se considera que as pessoas com deficiência estão alijadas deste progresso, porque estas tecnologias são foram projetadas de forma inclusiva, pensando nas diferentes necessidades comunicacionais de cada perfil de usuário, percebe-se uma grande injustiça. Bruno e Nascimento (2019, p. 10) afirmam que, para as pessoas cegas ou com baixa visão, ser "usuário das tecnologias é um fator que compõe a identidade desses sujeitos, os qualificam e os afirmam positivamente, quando colaboram para que sejam indivíduos produtivos, inseridos e participativos em diferentes grupos socioculturais."

Os parâmetros que definem se uma pessoa tem baixa visão têm pequenas diferenças em relação à acuidade visual. Neste artigo, usa-se a definição da WHO [200-]: "uma condição que resulta numa acuidade visual de menos de 6/181, no olho de melhor visão, considerando a melhor correção óptica possível". Scheiman et al. (2006) indicam que esta acuidade visual é insuficiente para ler um folheto, e que pessoas com acuidade visual de 6/60 mal conseguem ler manchetes de jornal. A visão funcional em pessoas com baixa visão é classificada em três níveis: moderada, severa e profunda. Se não for estimulada, o campo de visão é progressivamente reduzido. Em se tratando do uso de tecnologias de informação, quando uma pessoa com baixa visão faz uso das ferramentas de acessibilidade projetadas para usuários cegos, ela não estimula sua visão funcional, o que lhe causará uma diminuição da sua capacidade visual.

O censo brasileiro de 2010 indicou que quase $24 \%$ da população afirma possuir alguma deficiência, sendo que 3,5\% da população apresenta deficiência visual severa, caracterizando 6,5 milhões de pessoas. Motivados por este dado, que indica a importância da

1 Ambiente Virtual de Aprendizagem. acessibilidade em ferramentas educacionais digitais como os AVAs, ${ }^{1}$ o objetivo nesta pesquisa foi apresentar um guia com diretrizes de usabilidade e acessibilidade para o design de Avas, voltados a usuários com baixa visão. Segundo Perry et al. (2018), diretrizes são "recomendação não obrigatórias porém úteis, pois resumem um amplo campo de conhecimento - tanto empírico como científico - e tem como benefício aumentar a produtividade, reduzir arbitrariedade em decisões projetuais e reduzir tempo de treinamento". 


\section{Acessibilidade de tecnologias digitais}

2 World Wide Web Consortium

3 Authoring Tool Accessibility Guidelines, https://www. w3.org/WAl/standardsguidelines/atag/

4 User Agent Accessibility Guidelines, https://www. w3.org/WAl/standardsguidelines/uaag/

5 Accessibility Standards, https://www.w3.org/WAl/ standards-guidelines/

6 Web Accessibility Initiative - Accessible Rich Internet Applications, https:// www.w3.org/WAl/ standards-guidelines/aria/
À medida que o acesso à internet se torna mais disponível e veloz, aumenta a necessidade de suporte à usabilidade e à acessibilidade, pois esta é a melhor forma de assegurar a inclusão de diferentes usuários (ABOU-ZAHRA; BREWER; HENRY, 2012; TANAKA; ROCHA, 2011). Acessibilidade é uma forma de permitir que pessoas com deficiências participem igualitariamente na internet, sendo essencial para desenvolvedores e empresas que desejam criar web sites inclusivos, representando inclusive um modelo de negócios viável, pois as boas práticas para desenvolvimento de web sites acessíveis se sobrepõem com várias outras. Segundo a Convenção dos Direitos das Pessoas com Deficiências da ONU (2016, p. 9), acessibilidade significa "permitir que pessoas com deficiência vivam de forma independente e participem de forma completa em todos os aspectos da vida".

Schmutz et al. (2017) por exemplo, pediram que 110 pessoas, metade com deficiências e metade sem, avaliassem web sites projetados com e sem conformidade com diretrizes de acessibilidade para web. $O$ resultado foi que mesmo para as pessoas sem deficiências, os web sites projetados em conformidade com diretrizes de acessibilidade se mostraram mais fáceis de usar e mais interativos. Coursaris e Kim (2011), ao revisar 100 pesquisas sobre usabilidade para aplicações móveis, alertam que a Acessibilidade é uma das áreas de pesquisa menos contemplada, tendo sido alvo de apenas 2 pesquisas.

Segundo Cooper (2016), o w3 $\mathrm{C}^{2}$ tem três conjuntos complementares de diretrizes voltadas à acessibilidade: para ferramentas de autoria $\left(\mathrm{ATAG}^{3}\right)$; para agentes com o usuário ( $\left.\mathrm{UAAG}^{4}\right)$; para acessibilidade de conteúdo web $\left(\mathrm{WCAG}^{5}\right)$. Segundo o autor, as diretrizes em sua versão 2.0 foram projetadas de forma independente dos navegadores e linguagens de programação, para que pudessem ser implementadas da forma mais conveniente por todos. Contudo, o desenvolvimento tecnológico evidenciou a necessidade de atualização. Um exemplo é o padrão (WAI-ARIA ${ }^{6}$ ) do W3C, motivado pelo surgimento de novas "tags" do HTML5, que podem ser desenvolvidas usando "tags" cujo conteúdo se modifica conforme certos parâmetros, sinalizando esta mudança de estado para usuários de leitores de texto. A versão das diretrizes WCAG em 2020 é a 2.1. Segundo Souza et al. (2019), esta versão "contém 17 novos itens, que atendem a uma gama ampla de necessidades específicas: de aprendizagem (3), baixa visão (4), fala (2), vestibular (1) motora (6), cegueira (1)". Ferati et al. (2016) fazem uma afirmação pertinente sobre a aderência a estas diretrizes: mesmo que um web site atenda todas as diretrizes a experiência pode ser ruim se um leitor de tela não estiver disponível no idioma do usuário. 
2.1 Acessibilidade de tecnologias digitais voltadas para pessoas com baixa visão

Segundo Agner (2009), desenvolvedores web experimentam dificuldade em escolher quais recomendações de acessibilidade atendem cada perfil de usuário com deficiência. De acordo com Oliveira et al. (2012), como resultado, os desenvolvedores acabam por abandonar a busca e adaptam diretrizes conhecidas às necessidades de seus projetos, o que pode comprometer o atendimento a estes usuários com necessidades tão específicas.

Uma busca pela string < accessibility AND ("low vision" OR "visual impairment" OR "visually impaired") AND usability> no Scopus, entre 2000 e 2021, retornou 354 itens, entre artigos de periódicos, de

7 https://www.bibliometrix. org/ conferências e capítulos de livro. Usando a biblioteca bibliometrix, ${ }^{7}$ pode-se constatar um aumento consistente nas publicações ano a ano, como mostra a Figura 1.

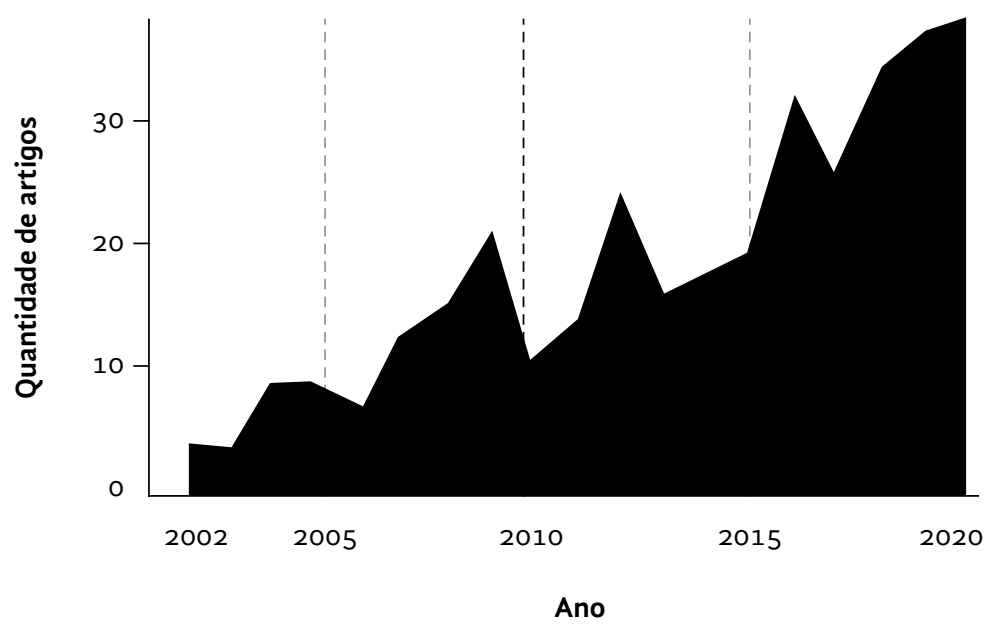

Figura 1 Produção científica anual.

Esse é um indicativo importante de que as necessidades das pessoas com baixa visão está chamando a atenção de pesquisadores.

\section{Recomendações de design para ambientes digitais para pessoas com baixa visão}

Foram encontradas várias pesquisas que fizeram recomendações voltadas para determinado público ou contexto (na internet), como por exemplo $\mathrm{Ng}$ (2017), que sugere algumas diretrizes para acessibilidade web sites de bibliotecas. Entre as sugestões da autora lista-se dicas para: escrever para a internet; redação de headings (cabeçalhos) e links; descrição de conteúdo audiovisual, de acordo com sua função; contraste de cores e estilos de textos. 
Pesquisas como a de Capovilla e Hubwieser (2013) - que desenvolveram e avaliaram um método para ensino de softwares de planilhas (i.e., MS Excel e Libre Calc) usando apenas os recursos de hardware (em notebooks) e software nativos, demonstraram que é possível atender estudantes com baixa visão - indicam que a inclusão deste público é possível. Taylor (2016) também fez algumas recomendações, com base em sua experiência com alunos com baixa visão, para design de materiais instrucionais para classes presenciais que atendam este perfil. A forma de reunir estas recomendações foi entrevistar alguns alunos no início do período letivo e tentar, à medida que o período progredia, manter contato próximo (inclusive com aulas individualizadas) e adaptar os materiais às necessidades destes alunos. Ainda que não tenha sido um método bem estruturado e com boa confiabilidade, o relato de Taylor indica um esforço genuíno para oferecer o melhor que estava a seu alcance. As recomendações que ele fez estão resumidas na lista abaixo:

- Certificar-se que os materiais instrucionais são compatíveis com as ferramentas de acessibilidade mais comuns (leitor de braile, leitor de tela e aumentadores de tela).

- Reconhecer as diversas necessidades comunicacionais, oferecendo sempre que possível diversos formatos. Lembrar que imagens, por exemplo, não são lidas pelos leitores de tela e que Power Points são pouco compatíveis com estes leitores.

- Estar preparado para que os tempos de realização de cada tarefa variem entre os estudantes.

- Planejar as aulas de forma previsível, eliminando complexidade e carga mental desnecessária.

- Incentivar a interação entre os estudantes, promovendo uma atmosfera colaborativa.

- Considerar que, ainda que o contexto das recomendações de Taylor seja diferente, elas podem ser importantes para esta pesquisa.

\section{Metodologia}

A metodologia para a elaboração das diretrizes baseia-se na pesquisa com usuários, ponderadas pela opinião de especialistas - dois atores centrais em métodos de projeto na área de Interação Humano-Computador. Considerou-se necessário inserir usuários representativos do público-alvo não apenas para ampliar a visão dos pesquisadores sobre as lacunas dos AVAs em relação à acessibilidade, mas porque não há como "colocar-se no lugar" de uma pessoa com baixa visão. Contudo, as visões a respeito da acessibilidade dos usuários não são informadas por conhecimentos técnicos, e por isso os especialistas nas áreas relacionadas à pesquisa também foram ouvidos. 
Perry et al. (2018) apontam que há três formas de realizar o levantamento de diretrizes: (1) revisão sistematizada de literatura; (2) revisão sistematizada de literatura ponderada com a opinião de usuários; (3) design centrado no usuário, sem revisão de literatura. Os dois primeiros métodos têm como objetivo minimizar os vieses dos pesquisadores, enquanto maximizam a reprodutibilidade (confiabilidade) da pesquisa. O método usado nesta pesquisa, portanto, enquadra-se no terceiro tipo, tendo a desvantagem de ser suscetível ao viés dos participantes e ao contexto da pesquisa. Perry et al. (2018), contudo, também apontam que os métodos que incluem revisão de literatura e excluem a opinião dos especialistas também tem desvantagens. Os autores concluíram que a eliminação dos vieses dos pesquisadores que estes métodos proporcionam impedem que a opinião dos especialistas seja utilizada para ponderar os resultados, de forma que o conjunto de diretrizes resultantes pode ser incompleto ou conter incoerências.

O método de elaboração de diretrizes tem três etapas consecutivas, como mostra a Figura 2. Participaram doze pessoas com baixa visão, sendo que quatro possuíam grau moderado, quatro possuíam grau severo, dois possuíam grau profundo e dois eram totalmente cegos. A faixa etária era bastante ampla, indo de 23 a 55 anos de idade, com média de 36 anos. Vinham de diversas áreas de formação: administração, direito, artes, jornalismo, letras, educação, história, geologia; sendo todos com formação superior completa. Também participaram três especialistas em usabilidade e acessibilidade (sendo que a primeira autora é um deles), e cinco desenvolvedores (programadores) e designers de interface.

A primeira etapa tem como objetivo compreender elementos importantes do contexto da pesquisa. Através de um Questionário (Apêndice 1), perguntou-se aos sujeitos de pesquisa, seus diagnósticos

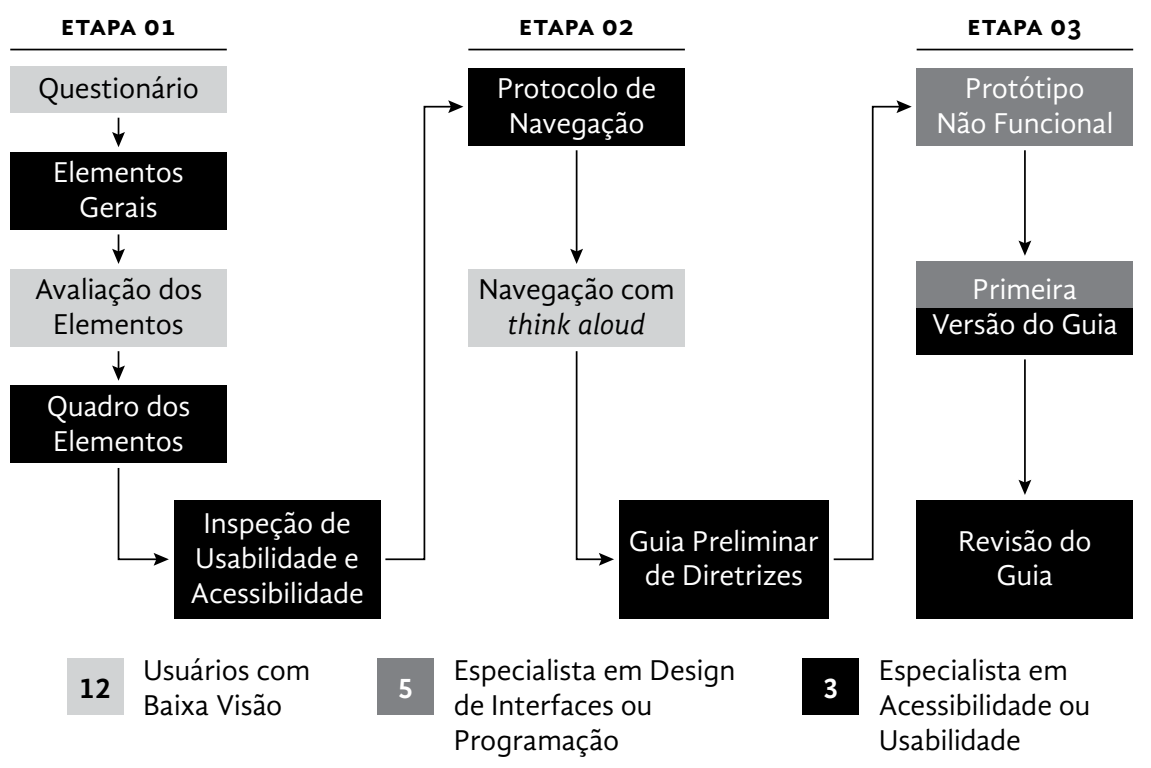

Figura 2 Metodologia proposta. Fonte: Autores. 
de baixa visão e visão funcional, idade e escolaridade, hábitos e dificuldades no uso da internet, assim como experiência com AVAs.

O resultado deste questionário é a identificação dos Elementos Gerais. De posse desta lista, os sujeitos de pesquisa avaliam os elementos/componentes de interação de acordo com sua relevância (Avaliação dos Elementos), gerando o Quadro dos Elementos, que serão inspecionados pelos especialistas em acessibilidade e usabilidade, na etapa Inspeção de Usabilidade e Acessibilidade, que marca a transição da primeira para a segunda etapa. Todas as etapas que envolvem avaliação de usabilidade e elaboração de protótipos foram apoiadas nas Técnicas de Inspeção de Usabilidade de Rocha e Baranauskas (2003) e nas Heurísticas de Usabilidade de Nielsen (1994).

O resultado desta inspeção informa a elaboração de um Protocolo de Navegação, que contém tarefas para os sujeitos de pesquisa, usando o método de "pensar em voz alta" (think aloud). Como resultado, foi elaborado um Guia Preliminar de Diretrizes, que foram avaliadas pelo conjunto de especialistas em acessibilidade, programação e design, que as utilizaram como base para construção de um Protótipo Não Funcional. Ao final, estes profissionais ajudaram a elaborar a Primeira Versão do Guia de Diretrizes, que foi revisada pelos autores deste artigo, resultando na Versão Revisada do Guia.

Os participantes da pesquisa usaram um tablet Samsung com sistema operacional Android, com os recursos de acessibilidade embarcados do equipamento. Escolheu-se usar um tablet por permitir mobilidade e para ter certeza que todos os usuários teriam acesso às mesmas configurações. O Moodle foi o AvA escolhido como objeto de análise, pois é o mais conhecido pelos participantes. Contudo, isso não significa que as conclusões desta pesquisa estejam restritas ao Moodle, pois as funções dos diversos Avas guardam alguma semelhança. Calvo et al. (2012) fizeram uma avaliação de acessibilidade do Moodle, voltada para o público com baixa visão, com base nas diretrizes do W3C ATAG 2.0 e WCAG 2.o. As avaliações foram realizadas com um usuário simulando cegueira e um especialista. Essa avaliação foi feita em 2012, com uma versão muito antiga do Moodle (1.9), que não havia incorporado diversas ferramentas de acessibilidade. À medida que as diretrizes de acessibilidade evoluíram, o Moodle foi sendo melhorado de forma que em 2019, Souza et al. (2019) realizaram uma avaliação automatizada da versão 2.9 do Moodle, usando duas ferramentas, e constataram que os problemas identificados por Calvo et al. (2012) não estão mais presentes.

\section{Resultados}

De acordo com o Questionário respondido, de uma forma geral, os sujeitos de pesquisa relataram que os AVAs possuem os seguintes problemas: contrastes inadequados, navegação inconsistente, layout poluído, falta de recursos de acessibilidade, falta de oferta de conteúdo textual. 
Em seguida, foram listados os Elementos Gerais, que são os componentes de interface mais frequentes. e 3 sujeitos de pesquisa com disponibilidade no momento, e autonomia para navegar sem o uso de programa de voz, mais de 18 anos e com visão funcional entre moderada à profunda navegaram pelo AVA, avaliando a lista dos Elementos Gerais de acordo com a importância percebida, usando uma escala Likert de 5 pontos. Os elementos gerais cuja importância somou menos de 10 pontos foram removidos, pois como a nota máxima é 15 $(5 \times 3), 10$ representa $2 / 3$ da nota máxima. Como resultado, chegou-se ao Quadro de Elementos, com 23 elementos gerais agrupados em 6 categorias (Quadro 1).

Quadro 1 Quadro dos Elementos da seção Perfil do Usuário do Ava Moodle, identificados como mais importantes pelos usuários com baixa visão.

\begin{tabular}{llll}
\hline & \multicolumn{2}{c}{ Elementos Gerais } \\
\hline Bloco de identidade & Título da pesquisa & Recursos de & Legenda \\
& Cabeçalho & acessibilidade & Texto descritivo \\
& Título de seções & & Texto alternativo \\
& Título para blocos & & Ampliação \\
& Rodapé & Contraste adaptado \\
& Endereço eletrônico & & P\&B \\
& & & Conteúdo sem estilo \\
& & Cursor piscante \\
\hline Bloco de navegação & Formulário de busca & Recursos de & Ícones e figuras \\
& Campo login/logout & Informação & Formulários \\
& Suporte aos usuários & & Link com ícone \\
& Menu de navegação & & \\
& Links & Cores \\
\hline Bloco de conteúdo & Seção informacional & Atributos & Tipografia \\
& Texto & & Blocos \\
\hline & Instrução textual & &
\end{tabular}

Na transição para a segunda etapa da metodologia, três especialistas avaliaram a usabilidade e a acessibilidade dos componentes identificados no Quadro 1, usando as 10 heurísticas de usabilidade de Nielsen (1994) e as diretrizes WCAG 2.0, MWBP 1.0 e MWABP (etapa Inspeção de Usabilidade e Acessibilidade). O resultado foi tabulado no Quadro 2, que apresenta os problemas observados divididos em 10 categorias.

Em decorrência desta avaliação, elaborou o Protocolo de Navegação, utilizado no Ensaio de Interação com think aloud por seis dos sujeitos desta pesquisa (três com visão funcional moderada, dois com severa e um com profunda) e que continha 24 tarefas de navegação direcionadas aos problemas identificados na etapa anterior. Devido à grande variabilidade dos perfis (grau de baixa visão e familiaridade com o AVA), o tempo médio de execução da tarefa não representa 
Quadro 2 Categorias de problemas e exemplos de ocorrências

\begin{tabular}{ll}
\hline Categoria & Problema observado \\
\hline Contraste & $\begin{array}{l}\text { Imagens, elementos interativos e texto com contraste inadequado } \\
\text { com o fundo. }\end{array}$ \\
\hline Ampliação & $\begin{array}{l}\text { Imagens ampliadas têm baixa resolução } \\
\text { Botões sobrepostos a outros elementos. } \\
\text { Elementos que não podem ser ampliados }\end{array}$ \\
\hline Links de navegação & $\begin{array}{l}\text { URL extensas, expressões longas e pouco conhecidas, baixo } \\
\text { contraste com o fundo, falta de diferenciação com o texto }\end{array}$ \\
\hline Vocabulário & Expressões pouco conhecidas \\
\hline Navegação & $\begin{array}{l}\text { Falta de feedback. } \\
\text { Falta de destaque para informações importantes. }\end{array}$ \\
\hline Feedback & Caixas de alerta que abrem fora do centro da tela \\
\hline Layout & $\begin{array}{l}\text { Informações repetidas desnecessariamente } \\
\text { Campo e link de busca deslocado de centro da tela } \\
\text { Ícones com muitos detalhes }\end{array}$ \\
\hline Prevenção de erros & $\begin{array}{l}\text { Falta de testes com diferentes perfis de usuários impossibilita } \\
\text { identificar as situações que poderiam ser evitadas }\end{array}$ \\
\hline Controle do usuário & $\begin{array}{l}\text { Dificuldade para identificar elementos da interface, interferindo na } \\
\text { capacidade de tomada de decisão. }\end{array}$ \\
\hline Ações que não têm o resultado esperado.
\end{tabular}

uma medida acurada. "Descreva os ícones que estão junto ao menu de navegação" e "Aponte onde está escrito a palavra acadêmico" são exemplos destas tarefas. Pediu-se que, durante a navegação, os usuários "pensassem em voz alta", comentando suas dificuldades, dando opiniões e relatando a experiência que estavam tendo. Estas sessões de navegação foram feitas individualmente, usando o mesmo tablet empregado nas etapas anteriores. Os sujeitos da pesquisa não conseguiram realizar algumas das tarefas de navegação em função de características da interface, como por exemplo: contrastes inadequados e imagens aumentadas que se sobrepunham ao texto.

A partir da transcrição dos relatos dos sujeitos de pesquisa, a primeira autora elaborou um Conjunto Preliminar de Diretrizes, com 42 diretrizes. Para a redação destas diretrizes, considerou-se os Requisitos de Aceitação de Tanaka e Rocha (2011): objetivos claros, redação simples, fácil de aprender como usar, fácil identificar o uso e fácil em aplicar. Após utilização destes requisitos de aceitação, verificou-se que 3 diretrizes não estavam adequadas, e elas foram reescritas.

A terceira e última etapa da metodologia consistiu na prototipagem da tela de login para o Moodle pelos cinco especialistas em design de interfaces e programação, com o objetivo de avaliar as diretrizes preliminares. De acordo com Santa Rosa e Moraes (2008), esta técnica 
é interativa e permite geração e avaliação rápida de alternativas, o que permite testar rapidamente a aderência de cada diretriz. Como referência, deixou-se à disposição dos desenvolvedores o tablet com o Moodle aberto em uma janela do navegador, bem como as diretrizes do guia preliminar impressas. Havia também um quadro branco, que poderia ser usado como apoio para elaboração do protótipo, papel e canetas. Este processo durou cerca de duas horas.

Os especialistas consideraram que 39 das 42 diretrizes estavam adequadas (ainda que algumas tiveram que ser reescritas) e 1 diretriz foi adicionada. Os desenvolvedores sugeriram dividir as diretrizes entre recomendações de "Design" e de "Programação". A etapa final foi a revisão da Primeira Versão do Guia pelos autores desta pesquisa, para a redação da Versão Final. Nesta etapa, duas especialistas buscaram eliminar redundâncias e revisaram a linguagem, o que resultou no conjunto de 23 diretrizes, onde o processo de filtragem impediu a distinção entre diretrizes de Design e de Programação, mostrando o conjunto final na seção seguinte.

\section{Diretrizes de acessibilidade para AVAS, voltadas a estudantes com baixa visão}

1. Fornecer destaque para o título da página, para que os usuários com baixa visão sempre saibam em que página estão.

2. Manter o padrão de páginas consistente, pois a redundância e a previsibilidade ajudam o usuário com baixa visão a se localizarem.

3. Posicionar os elementos (por exemplo: blocos, textos e imagens) de forma que, ao ampliar a página, os espaços em branco não fiquem tão evidentes a ponto de ocupar muito espaço na tela, o que faria com que o usuário perca a referência de onde está.

4. Para facilitar a navegação, criar blocos de conteúdo em colunas organizadas e limpas (sem desenhos, sem texturas), para que se mantenham quando ampliadas.

5. Quando pertinente, organize as informações por ordem cronológica crescente, pois isso acelera a busca, como em, por exemplo, fóruns e mensagens.

6. Escolher cores que tenham bastante contraste entre si.

7. Criar ícones com poucos detalhes e alto-contraste. Se o ícone tiver texto, usar fonte de tamanho médio, ou seja, equivalentes ao tamanho do atributo css "font-size: medium".

8. Colocar no topo da página o menu principal. Se possível, usar menu contextual.

9. Colocar o Perfil do Usuário e os links para Ajuda e Suporte ao Usuário em local de destaque

10. Posicionar campo de busca no topo da página, ou junto aos links principais. 
11. Fornecer um menu tipo breadcrumbs (migalhas de pão), que permite que o usuário com baixa visão consiga voltar várias páginas com apenas um clique.

12. Fornecer mensagens de erro e outras ações (como esperar o carregamento de um arquivo) no centro da página e permitir que o usuário decida quando quer fechar esta caixa de diálogo.

13. Se for usar janela pop up, usar áudio ou animações para chamar atenção.

14. As ferramentas de acessibilidade devem permitir ampliação de texto até pelo menos até $200 \%$.

15. Permitir a reconfiguração da acessibilidade por página, pois as páginas de um mesmo web site podem precisar de diferentes configurações.

16. Disponibilizar ferramentas de acessibilidade em todas as páginas.

17. As ferramentas de acessibilidade devem oferecer opções de contrastes predefinidos, mas também devem permitir a configuração de contrastes personalizados, pois as necessidades o usuário com baixa visão são bastante variadas.

18. Para melhorar a acessibilidade em dispositivos móveis, permitir formas de ampliação maiores que as que são disponibilizadas pelas configurações de acessibilidade dos dispositivos, e que não dependam apenas do movimento de "pinça".

19. Realizar avaliação automatizada de acessibilidade, para identificar situações de não conformidade com as diretrizes do WCAG.

20. Use unidades de tamanho relativa como "em" sempre que possível, por exemplo, em fontes e margens.

21. Sempre colocar descrição nas imagens. Usar, além do atributo "alt", as tags "figure" e "figcaption" se possível.

22. Fornecer url encurtadas nos menus de navegação mais acessados.

23. Preferir sempre expressões comuns e objetivas para redigir textos e links.

\section{Conclusão}

Ainda que a maioria das diretrizes listadas nesta pesquisa não representem, em si, novidade em termos de design de interfaces, o fato de elas terem sido agrupadas em torno das necessidades dos usuários com baixa visão representa um avanço, pois facilita o uso destas diretrizes por desenvolvedores que não são especialistas no assunto. Determinar quais as recomendações que melhor contemplam o usuário com baixa visão é um processo desgastante e desafiador para o desenvolvedor, pois requer um conhecimento aprofundado sobre as necessidades destes usuários, desde suas características, dificuldades, expectativas, demandas, entre outros. Esta compreensão 
demanda tempo e não garante que o desenvolvedor identifique todas as diretrizes necessárias nesse caso e tampouco que as implemente corretamente.

Apesar de existirem diversas diretrizes de acessibilidade com linguagem simples e clara, é difícil identificar para quais situações de uso são mais apropriadas. Essas recomendações trazem consigo uma mesma característica, que é possibilitar que uma interface seja utilizada por uma quantidade maior de usuários, quer eles tenham dificuldades ou não. Na revisão da literatura feita neste artigo, não foram encontrados conjuntos de diretrizes voltadas para este usuário específico. Por este motivo, o guia de diretrizes resultante desta pesquisa é considerado mais fácil de implementar pelos desenvolvedores, devido tanto à participação dos usuários com baixa visão como dos especialistas em usabilidade e acessibilidade, durante as etapas da metodologia proposta pela autora.

Como sugestões para estudos futuros, propõe-se que seja ampliado o conjunto de diretrizes de forma que possam ser incluídos outros perfis de usuários, tais como pessoas com daltonismo severo. Além disso, propõe-se desenvolver interfaces do usuário de diferentes AVAs, usando o guia de diretrizes para usuários com Baixa Visão a fim de avaliá-lo empiricamente.

\section{Referências}

ABOU-ZAHRA, S.; BREWER, J.; HENRY, S. L. Essential components of mobile web accessibility. In: INTERNATIONAL CROSS-DISCIPLINARY CONFERENCE ON WEB ACCessibility, 10., 2013, Rio de Janeiro. Anais [...]. New York: Association for Computing Machinery, 2013, p. 1-4. DOI: https://doi.org/10.1145/ 2461121.2461138

AGNER, L. Ergodesign e arquitetura de informação: trabalhando com o usuário. 2. ed. Rio de Janeiro: Quarteto, 2009.

CALVO, R.; IGLESIAS, A.; MORENO, L. Is moodle accessible for visually impaired people? In: FILIPE, J.; CORDEIRO, J. (orgs.). Web information systems and technologies (WEBIST 2011): lecture notes in business information processing, v. 101. Heidelberg: Springer, 2012. p. 207-220. DoI: https://doi. org/10.1007/978-3-642-28082-5-1

Bruno, M. M. G.; NAScimento, R. A. L. Política de acessibilidade: o que dizem as pessoas com deficiência visual. Educação \& Realidade, Porto Alegre, v. 44, n. 1, p. 1-15, 2019. DOI: https://doi.org/10.1590/2175-623684848

CAPOVILLA, D.; HUBWIESER, P. Teaching spreadsheets to visually-impaired students in an environment similar to a mainstream class. In: ACM CONFERENCE ON INNOVATION AND TECHNOLOGY IN COMPUTER SCIENCE EdUCATion, 18., 2013, Canterbury. Proceedings [...]. New York: Association for Computing Machinery. 2013, p. 99-104. DOI: 10.1145/2462476.2462477 COOPER, M. Web Accessibility Guidelines for the 2020s. In: INTERNATIONAL Web fOR ALl CONFERENCE, 13., 2016, Montreal. Proceedings [...]. 
New York: Association for Computing Machinery. 2016, p. 1-4. DOI: 10.1145/2899475.2899492

COURSARIS, C. K.; KIM, D. J. A meta-analytical review of empirical mobile usability studies. Journal of Usability Studies, [S.1.], v. 6, n. 3, p. 117-171, 2011. NG, C. A practical guide to improving web accessibility. Journal of Library User Experience, [S.1.], v. 1, n. 17, 2017. DOI: 10.3998/weave.12535642.0001.701 NIELSEN, J.; MACK, R. L. Usability inspection methods. New York: John Wiley \& Sons, 1994.

Oliveira, D. H. D. et al. Prototipação de interfaces de aplicativos para dispositivos móveis: estado da arte e desafios de IHC. In: BRAZILIAN SYMPOSIUM ON human factors in Computing Systems, 11., 2012, Cuiabá. Proceedings [...]. Porto Alegre: Brazilian Computer Society. 2012, p. 315-324.

PERRY, G. T.; POSSATTI, G. M.; SILVA, R. P. Guidelines for ebook design. InfoDesign, São Paulo, v. 15, n. 2, p. 197-213, 2018. Disponível em: https://infodesign.org. br/infodesign/article/view/651. Acesso em: 31 jul. 2020.

ROCHA, H. V. R.; BARANAUSKAS, M. C. Design e avaliação de interfaces humanocomputador. Campinas: NIED/UNICAMP, 2003. Disponível em: https://www. nied.unicamp.br/biblioteca/design-e-avaliacao-de-interfaces-humanocomputador/. Acesso em: 31 jul. 2020.

SCHEIMAN, M.; SCHEIMAN, M.; WHITTAKER, S. G. Low vision rehabilitation: a practical guide for occupational therapists. New Jersey: Slack Incorporated, 2006.

SCHMUTZ, S.; SONDEREGGER, A.; SAUER, J. Implementing recommendations from web accessibility guidelines: a comparative study of nondisabled and users with visual impairments. Human Factors, [S.1.], v. 59, n. 6, p. 956-972, 2017. DOI: 10.1177/0018720817708397.

SOUZA, N.; CARDOSO, E.; PERRY, G. T. Limitações da avaliação automatizada de acessibilidade em uma plataforma de moocs: estudo de caso de uma plataforma brasileira. Revista Brasileira de Educação Especial, Bauru, v. 25, n. 4 , p. 603-616, 2019. DOI: 10.1590/s1413-65382519000400005

TANAKA, E. H.; ROCHA, H. v. Evaluation of web accessibility tools. In: BRAZILIAN SYMPOSIUM ON HUMAN FACTORS IN COMPUTING SYSTEMS, 10.; LATIN AMERICAN Conference on human-Computer interaction, 5., 2011, Porto de Galinhas. Proceedings [...]. Porto Alegre: Brazilian Computer Society. 2011, p. 272-279. TAYLOR, M. A. Improving accessibility for students with visual disabilities in the technology-rich classroom. PS: Political Science \& Politics, [S.l.], v. 49, n. 1, p. 122-127, 2016. DOI: 10.1017/S1049096515001134

UNITED NATIONS. Convention on the rights of persons with disabilities and optional protocol. 2006. Disponível em: https://www.un.org/disabilities/ documents/convention/convoptprot-e.pdf. Acesso em: 8 fev. 2021.

WHO. Blindness and vision impairment prevention. Disponível em: https:// www.who.int/news-room/fact-sheets/detail/blindness-and-visualimpairment. Acesso em: 31 jul. 2020. 


\section{Sobre os autores}

\section{Cinthia Kulpa}

cinthia.kulpa@gmail.com

Professora da Faculdade de Arquitetura - Curso de Design - UFRGS,

Universidade Federal do Rio Grande do Sul

R. Sarmento Leite, 320 - Centro Histórico, Porto Alegre, RS, 90050-170

\section{Gabriela Trindade Perry}

gabriela.perry@ufrgs.br

Professora do PPGIE - Ufrgs, Programa do Pós Graduação em

Informática na Educação, Universidade Federal do Rio Grande do Sul Av. Paulo Gama, 110 - 329 - Farroupilha, Porto Alegre, Rs, 90040-060

\section{Fernando Gonçalves Amaral}

amaral@producao.ufrgs.br

Professor do PPGEP - UFRGs, Programa do Pós Graduação em

Engenharia de Produção, Universidade Federal do Rio Grande do Sul Av. Osvaldo Aranha, 99 - 50 Andar - Bom Fim, Porto Alegre, RS, 90.035-190

Artigo recebido em/Submission date: 4/8/2020

Artigo aprovado em/Approvement date: 3/4/2021 


\section{Apêndice 1}

\section{Questionário prévio}

A princípio tenho que delimitar a pesquisa entendendo quais os usuários com Baixa Visão serão considerados sujeitos da pesquisa e quais os Ambientes Virtuais de Aprendizagem serão utilizados nos testes que faremos com estes sujeitos. Apesar de saber que são muitos os diagnósticos que determinam a Baixa Visão pretendo englobar a maioria dos usuários, de acordo com suas possibilidades no uso dos Ambientes Virtuais de Aprendizagem, no tablet. Portanto, é importante para esta pesquisa, sua colaboração.

Usuário nº: [preenchido pela pesquisadora]

1. Qual o seu diagnóstico de Baixa Visão?

2. como é sua visão funcional (conte-me um breve histórico das facilidades e possibilidades com sua visão funcional).

3. Preciso saber mais um pouco sobre você: idade, escolaridade, profissão.

4. Preciso saber se você já utilizou um Ambiente Virtual de Aprendizagem. Qual?

5. Você consegue (e por quanto tempo) navegar em um Ambiente Virtual de Aprendizagem sem o uso de programas de voz?

6. Quais são os Ambientes Virtuais de Aprendizagem que você gostaria muito de navegar com mais facilidade (se possível, cite alguns exemplos).

7. Quais os problemas que você percebe nestes Ambientes Virtuais de Aprendizagem indicados quando utiliza-os (por gentileza: descrever os problemas detalhadamente se possível)?

8. Você já navegou em um Ambiente Virtual de Aprendizagem utilizando o tablet?

9. Você já utilizou tablet para outros fins? Quais? 